\title{
Assistência do enfermeiro diante do câncer de mama na estratégia da família
}

\author{
Nurse assistance in the face of breast cancer in the family strategy \\ Asistencia de enfermería ante el cáncer de mama en la estrategia familiar
}

Recebido: 01/07/2021 | Revisado: 09/07/2021 | Aceito: 12/07/2021 | Publicado: 22/07/2021

Brenda Cardoso Arruda Ferreira

ORCID: https://orcid.org/0000-0003-4226-2802

Universidade Castelo Branco, Brasil

E-mail: brenda_arruda_@hotmail.com

Thaís Araújo Vianna

ORCID: https://orcid.org/0000-0002-0892-5898 Universidade Castelo Branco, Brasil

E-mail: thais.pnk@gmail.com

Jamile Santana Dos Santos Barbosa

ORCID: https://orcid.org/0000-0003-1906-2095 Universidade Castelo Branco, Brasil E-mail: jammy_santana@hotmail.com

Mariana Keller Campos Lima

ORCID: https://orcid.org/0000-0003-0618-2784 Universidade Castelo Branco, Brasil E-mail: mari.keller@hotmail.com

Sandra Conceição Ribeiro Chícharo

ORCID: https://orcid.org/0000-0002-1487-0088 Universidade Castelo Branco, Brasil E-mail: sandracrchicharo@gmail.com

Lidiane Rossato Deckmann Nogueira

ORCID: https://orcid.org/0000-0002-5913-1334

Universidade Nossa Senhora de Fátima, Brasil E-mail: lidydeck@gmail.com

\begin{abstract}
Resumo
Objetivo: Analisar assistência do enfermeiro diante do câncer de mama na estratégia da família. Método: Trata-se de uma pesquisa qualitativa, descritiva de revisão integrativa de literatura nas bases de dados. A busca efetuou-se, através da Plataforma da Biblioteca Virtual em Saúde - BVS, utilizando as bases de dados Literatura Latino-Americana e do Caribe em Ciências da Saúde (LILACS), Medical Literature Analysis and Retrieval System Online (MEDLINE), Base de Dados de Enfermagem (BDENF), e por meio do Portal Google Acadêmico, aderindo-se através dos descritores: Cuidados de enfermagem; Câncer de Mama. Resultados: Diante dos resultados obtidos, inicialmente foram filtrados e selecionados um total de 78 artigos, disponíveis na íntegra, nos idiomas inglês e português, publicados a partir do ano de 2017. Desses, 4 encontravam-se duplicados e 49 foram excluídos pelo título e resumo, por não contemplarem a revisão proposta. Portanto, foram selecionados e incluídos nesta revisão um total de dez artigos. Ao analisar as publicações, verifica-se que os 25 artigos (100\%) foram publicados em revistas de enfermagem brasileiras. Discussão: Foram abordados os seguinstes temas: Câncer de mama e saúde pública, Reconfigurando velhas questões: câncer de mama e a estruturação do SUS, Fatores de risco, prevenção primária e secundária, detecção precoce do C.A de mama, O mal das senhoras e a mastectomia radical, Educação em saúde e o Câncer de mama, Câncer de mama e o papel do enfermeiro, Qualidade de vida e câncer de mama, Câncer de mama e Covid-19.
\end{abstract}

Palavras-chave: Cuidados de enfermagem; Câncer de mama.

\begin{abstract}
Objective: To analyze nursing care in the face of breast cancer in the family strategy. Method: This is a qualitative research, descriptive of an integrative literature review in databases. The search was carried out through the Virtual Health Library Platform - VHL, using the databases Latin American and Caribbean Literature in Health Sciences (LILACS), Medical Literature Analysis and Retrieval System Online (MEDLINE), Database Nursing Data (BDENF), and through the Academic Google Portal, adhering through the descriptors: Nursing care; Breast cancer. Results: Based on the results obtained, a total of 78 articles were initially filtered and selected, available in full, in English and Portuguese, published from 2017 onwards. Of these, 4 were duplicated and 49 were excluded by title and summary, for not contemplating the proposed revision. Therefore, a total of ten articles were selected and included in this review. When analyzing the publications, it appears that the 25 articles (100\%) were published in Brazilian nursing journals. Discussion: The following topics were addressed: Breast cancer and public health, Reconfiguring old issues: breast cancer and the structuring of the SUS, Risk factors, primary and secondary prevention, early detection of breast
\end{abstract}


cancer, Ladies' disease and radical mastectomy, Health education and breast cancer, Breast cancer and the role of the nurse, Quality of life and breast cancer, Breast cancer and Covid-19.

Keywords: Nursing care; Breast câncer.

\begin{abstract}
Resumen
Objetivo: Analizar el cuidado de enfermería ante el cáncer de mama en la estrategia familiar. Método: Se trata de una investigación cualitativa, descriptiva de una revisión integradora de la literatura en bases de datos. La búsqueda se realizó a través de la Plataforma de Biblioteca Virtual en Salud - BVS, utilizando las bases de datos Literatura Latinoamericana y del Caribe en Ciencias de la Salud (LILACS), Sistema de Análisis y Recuperación de Literatura Médica en Línea (MEDLINE), Base de Datos de Enfermería (BDENF), y a través del Portal académico de Google, utilizando los descriptores: Atención de enfermería; Cáncer de mama. Resultados: Con base en los resultados obtenidos, inicialmente se filtraron y seleccionaron un total de 78 artículos, disponibles íntegramente, en inglés y portugués, publicados a partir de 2017. De estos, 4 fueron duplicados y 49 fueron excluidos por título y resumen, por no contemplando la revisión propuesta. Por lo tanto, se seleccionaron e incluyeron un total de diez artículos en esta revisión. Al analizar las publicaciones, parece que los 25 artículos (100\%) fueron publicados en revistas brasileñas de enfermería. Discusión: Se abordaron los siguientes temas: Cáncer de mama y salud pública, Reconfiguración de viejos temas: cáncer de mama y la estructuración del SUS, Factores de riesgo, prevención primaria y secundaria, detección precoz del cáncer de mama, Enfermedad de la mujer y mastectomía radical, Educación para la salud y cáncer de mama, Cáncer de mama y el papel de la enfermera, Calidad de vida y cáncer de mama, Cáncer de mama y Covid-19.
\end{abstract}

Palabras clave: Cuidado de enfermera; Cáncer de mama.

\title{
1. Introdução
}

O câncer de mama é uma patologia neoplásica desenvolvida pela multiplicação de forma desordenada e descontrolada de células da mama, alterando a sua morfologia e se dividem rapidamente, podendo progredir para todo o organismo (Inácio et al.,2020).

O controle do câncer de mama tem sido eficaz quando a doença é descoberta precocemente, possibilitando o recurso terapêutico com mais chances de cura. Consideramos que $80 \%$ das descobertas são através do autoexame das mamas, mas esta estratégia não tem sido eficiente quando detectada, pois geralmente apresentam em fase avançada. Os métodos mais seguros e eficazes são o exame clínico e mamografia (Rodrigues et al.,2020).

O câncer de mama hoje é considerado um grande problema de saúde pública em todo o mundo, é o segundo tipo de neoplasia mais afetado nas mulheres perdendo apenas para o melanoma. É considerada uma doença temida entre as mulheres por acometer um órgão que identifica a feminilidade e a sexualidade. Apresenta uma incidência considerável a partir dos 40 anos e um aumento de até 10 vezes acima de 60 anos. Os homens também podem ser acometidos com uma proporção de $1 \%$ do total de casos da doença (Nunes et al., 2020).

A cura depende muito do tipo de tumor e do estágio em que se encontra a doença pois aqueles que são diagnosticados precocemente tem um resultado satisfatório ou proporciona melhores condições de sobrevida, por este motivo existe a necessidade de um controle para realizar exames sempre que necessário ou anualmente para identificar essas alterações iniciais proporcionando chances maiores de cura (Nunes et al., 2020).

O termo câncer refere-se às neoplasias ou tumores malignos, que são lesões expansivas formadas pelo aumento desordenado do número de células, que em geral têm crescimento rápido e muitas provocam perturbações homeostáticas graves que acabam levando o indivíduo à morte. $\mathrm{O}$ termo advém do grego (karkinos = caranguejo), sendo usado inicialmente por Galeno (aproximadamente 138 a 201 d.C.) ao se referir ao câncer de mama, no qual as veias superficiais do órgão se assemelhavam às patas de um caranguejo (Amaral et al., 2019).

Quanto à classificação, a quimioterapia pode ser neoadjuvante, geralmente adotada na terapêutica de tumores grandes, tendo assim o objetivo de reduzir o tumor, para facilitar a cirurgia e evitando que ela seja mais agressiva para facilitar cirurgias conservadoras. Já a quimioterapia adjuvante, se realiza após a cirurgia para a retirada de um tumor primário, visando à eliminação de um possível risco de recidiva, podendo reduzir o resurgimento e mortalidade (Coelho et al., 2017). 
Nesse contexto, a quimioterapia é, portanto, uma modalidade terapêutica de ação sistêmica, realizada por meio de administração de alta dosagem de substâncias, que, em sua maioria, são aplicados por via endovenosa, podendo também ser por via oral, intramuscular, subcutânea, tópica e intratecal, com o objetivo de aniquilar o crescimento e a divisão celular, das células tumorais (Brasil, 2018).

Segundo o Instituto Nacional do Câncer-INCA, o câncer de mama é uma doença resultante da multiplicação de células anormais da mama, formando assim um tumor com o potencial para invadir outros órgãos. Afirma também que quando a doença é diagnosticada no início, a maioria dos pacientes tem boa resposta ao tratamento (Ribeiro et al., 2020).

$\mathrm{O}$ exame físico é o principal método diagnóstico dessa patologia em abrangência a atenção primária à saúde. $\mathrm{O}$ primeiro sinal clínico tende a ser descoberto pelo próprio paciente, geralmente ou pela presença do nódulo na mama ou da ginecomastia. Os sinais e sintomas do câncer de mama masculino assemelham-se aos do feminino, o que dificulta a detecção precoce e a demora do homem em buscar os serviços de atenção primária à saúde (Ribeiro et al.,2020).

A Enfermagem, por acompanhar o paciente de forma mais próxima, está presente nos diferentes estágios e cenários, atuando desde a prevenção primária aos cuidados pós-diagnóstico e na reabilitação, prestando uma assistência holística. Ressalta-se ainda seu papel dentro da equipe multidisciplinar, na discussão dos casos, na tomada de decisões, na realização de planos de cuidados e na manutenção do conforto físico e emocional (Silva et al.,2021).

$\mathrm{O}$ tratamento do câncer causa sofrimento à mulher, afeta a sua autoimagem e a feminilidade, provocando um distanciamento dos padrões estéticos, além de deixá-la muitas vezes com pensamentos pessimistas, insegurança e medo de ter metástase, morte ou câncer reincidente. Percebe-se a importância dos cuidados de enfermagem, do acompanhamento efetivo e humanizado, com um olhar e atendimento holístico em todos os momentos (Inácio et al.,2020).

O câncer ainda é entendido pelas pessoas, em geral, como sinônimo de dor, morte e sofrimento. Nesta perspectiva, cabe à enfermagem identificar suas próprias concepções relativas ao câncer e estabelecer estratégias de enfrentamento, embasadas nas resoluções do COFEN 358/2009, 210/1998 e 211/1998 que os ampara, visando assim uma assistência adequada, humanizada e eficaz que possibilite minimizar o sofrimento de todos os envolvidos (Leite et al., 2021).

No momento do recebimento do diagnóstico de câncer de mama e das informações referentes à doença e tratamento, a mulher depara-se com a fragilidade da sua existência e a possibilidade da morte torna-se presente em seus pensamentos. Esse diagnóstico traz muitas mudanças na vida e rotina da paciente, pois gera um grande conflito emocional, passando por etapas que vão desde a negação até a aceitação do diagnóstico, uma vez que o câncer ainda é visto como uma doença incurável. O psicológico, não somente da paciente, mas também de toda a família torna-se vulnerável, predominando o sentimento do medo da experiência inesperada que irão vivenciar (Souza et al., 2020).

Desse modo, é de extrema importância que o enfermeiro atue em ações multidisciplinares desde a prevenção, diagnóstico e recuperação, visto que englobar propostas educativas favorece um maior conhecimento sobre o assunto. Para tanto, o enfermeiro deve ser capacitado a fim de compreender as atitudes e medos ao realizar o planejamento de ações pela melhor qualidade de vida dessas pacientes (Souza et al., 2020).

\section{Metodologia}

Trata-se de uma pesquisa qualitativa, descritiva de revisão integrativa de literatura nas bases de dados. Segundo Minayo, é descrito como método qualitativo aquele que pode ser definido e aplicado ao estudo da história, das relações, das representações, das crenças, das percepções e das opiniões, produtos das interpretações que os humanos fazem a respeito de como vivem, constroem seus artefatos e a si mesmos, sentem e pensam. 
A busca efetuou-se, através da Plataforma da Biblioteca Virtual em Saúde - BVS, utilizando as bases de dados Literatura Latino-Americana e do Caribe em Ciências da Saúde (LILACS), Medical Literature Analysis and Retrieval System Online (MEDLINE), Base de Dados de Enfermagem (BDENF), e por meio do Portal Google Acadêmico, aderindo-se através dos descritores: Cuidados de enfermagem; Câncer de Mama.

A revisão configura-se, portanto, como um tipo de revisão da literatura que reúne achados de estudos desenvolvidos mediante diferentes metodologias, permitindo aos revisores sintetizar resultados sem ferir a filiação epistemológica dos estudos empíricos incluídos. Para que esse processo concretize-se de maneira lógica, isenta de desatinos epistemológicos, a revisão requer que os revisores procedam à análise e à síntese dos dados primários de forma sistemática e rigorosa (Leite et al., 2021).

Esse instrumento de estudo requer um padrão de excelência quanto ao rigor metodológico para que seu produto possa trazer contribuições significativas para a ciência e para a prática clínica. A preservação deste padrão requer o uso de métodos que garantam a análise precisa, objetiva e completa do tema revisado; o suporte teórico para analisar resultados, métodos, sujeitos e variáveis dos estudos primários; a provisão de todas as informações contidas nos estudos revisados e não apenas os principais resultados, de modo a informar o leitor sem o sobrecarregar com informações desnecessárias (Silva et al., 2021).

O acompanhamento dos seguintes procedimentos, que devem ser desenvolvidos de forma crítica pelo revisor, foi sugerido: definir a questão ou o assunto da RI de forma clara; indicar hipóteses, que devem ser complementares à questão norteadora da revisão; descrever, de forma detalhada, os critérios de seleção dos estudos a serem incluídos na revisão; definir e descrever as características dos artigos analisados, etapa considerada núcleo da revisão integrativa (Leite et al., 2021).

\section{Resultados}

Diante dos resultados obtidos, inicialmente foram filtrados e selecionados um total de 78 artigos, disponíveis na íntegra, nos idiomas inglês e português, publicados a partir do ano de 2017. Desses, 4 encontravam-se duplicados e 49 foram excluídos pelo título e resumo, por não contemplarem a revisão proposta. Portanto, foram selecionados e incluídos nesta revisão um total de dez artigos. Ao analisar as publicações, verifica-se que os 25 artigos (100\%) foram publicados em revistas de enfermagem brasileiras.

\section{Discussão}

\section{Câncer de mama e saúde pública}

Apesar dos métodos diagnósticos serem ofertados pelo SUS, ainda que de forma insuficiente e desarticulada, o principal desafio em relação ao câncer de mama é o diagnóstico precoce4. A Atenção Primária à Saúde (APS), pela facilidade de acesso, de coordenação e por seu caráter longitudinal, apresenta potencialidades para interferir em algumas das condições que acompanham o adoecimento por câncer5. Para tal, demanda resolutividade a partir do apoio diagnóstico e terapêutico dos pontos especializados e hospitalares6, regulados a partir das necessidades de saúde dos territórios (Almeida et al., 2020).

Processos regulatórios em saúde como ferramenta de gestão apresentam potência para reduzir e minorar desigualdades, em especial quando envolvem entes públicos e privados. Em 2008, foi instituída a Política Nacional de Regulação do Sistema Único de Saúde, na qual a Regulação do Acesso ou Regulação Assistencial tem como objetivos a organização, o controle, o gerenciamento e a priorização do acesso e dos fluxos assistenciais no SUS. Essa dimensão contempla as ações de regulação médica e o estabelecimento de referências entre diferentes níveis de complexidade a partir de centrais de regulação e complexos reguladores, de abrangência municipal, regional ou estadual, segundo fluxos e protocolos pactuados (Melo et al.,2020). 


\section{Reconfigurando velhas questões: câncer de mama e a estruturação do SUS}

Apesar dos métodos diagnósticos serem ofertados pelo SUS, ainda que de forma insuficiente e desarticulada, o principal desafio em relação ao câncer de mama é o diagnóstico precoce. A Atenção Primária à Saúde (APS), pela facilidade de acesso, de coordenação e por seu caráter longitudinal, apresenta potencialidades para interferir em algumas das condições que acompanham o adoecimento por câncer. Para tal, demanda resolutividade a partir do apoio diagnóstico e terapêutico dos pontos especializados e hospitalares, regulados a partir das necessidades de saúde dos territórios (Almeida et al.,2020).

Processos regulatórios em saúde como ferramenta de gestão apresentam potência para reduzir e minorar desigualdades, em especial quando envolvem entes públicos e privados. Em 2008, foi instituída a Política Nacional de Regulação do Sistema Único de Saúde, na qual a Regulação do Acesso ou Regulação Assistencial tem como objetivos a organização, o controle, o gerenciamento e a priorização do acesso e dos fluxos assistenciais no SUS. Essa dimensão contempla as ações de regulação médica e o estabelecimento de referências entre diferentes níveis de complexidade a partir de centrais de regulação e complexos reguladores, de abrangência municipal, regional ou estadual, segundo fluxos e protocolos pactuados (Almeida et al.,2020).

No caso do câncer de mama, o cenário moldado pelo novo sistema de saúde e pelo desenvolvimento da medicina baseada em evidência e dos protocolos médicos permitiu a reconfiguração de questões já colocadas há muito tempo, entre as quais se destacam o uso de práticas terapêuticas conservadoras e as proposições sobre o rastreamento para câncer de mama no país. A combinação da mastectomia a outras técnicas terapêuticas, como a quimioterapia, a hormonioterapia e a radioterapia, permitiu um aprofundamento da crítica ao método halstediano e a aplicação de procedimentos menos agressivos. A preocupação com a qualidade de vida das pacientes ganhou mais atenção das instituições de assistência, com a atuação de profissionais da área psicológica, do serviço social e da enfermagem no cuidado às pacientes mastectomizadas (Bervian; GirardonPerlinni, 2006).

\section{Fatores de risco, prevenção primária e secundária, detecção precoce do C.A de mama}

$\mathrm{O}$ câncer de mama ocorre quando há uma proliferação exacerbada das células, ou seja, uma multiplicação rápida e desordenada celular que pode ocorrer por fatores ambientais e genéticos. Além disso, o estrogênio tem grande atuação no crescimento das células da mama, o que resulta em um potencial aumento de modificações genéticas, sendo assim, qualquer fator que aumente os níveis de estrogênio, consequentemente, aumenta o risco de desenvolvimento do câncer (Oliveira et al.,2019).

A detecção precoce do câncer de mama visa a identificar a doença em fase inicial, seja por meio do diagnóstico precoce, estratégia dirigida às mulheres com sinais e sintomas suspeitos da doença, ou do rastreamento mamográfico, exames de rotina em mulheres assintomáticas em faixa etária e periodicidade definidas (Santos et al.,2020).

Os fatores de risco que estão mais vinculados ao desenvolvimento do câncer de mama são a idade avançada - segundo fator de risco mais forte - as características reprodutivas, a história familiar e pessoal, os hábitos de vida e as influências ambientais. No entanto, o fator de risco mais importante é o gênero, já que no sexo feminino a doença tem uma maior frequência chegando à incidência de 100 a 150 vezes superior quando comparado com o sexo masculino, este fato é explicado pela quantidade superior de tecido mamário e exposição ao estrogênio endógeno nas mulheres (Costa et al.,2019).

A história pessoal e familiar tem influência quando há um ou mais componentes da família com menos de 50 anos de primeiro grau com câncer de mama, assim como câncer de mama bilateral ou câncer ovariano em um ou mais familiar de primeiro grau independentemente da idade, câncer de mama em componente familiar do sexo masculino e também câncer de mama e/ou doença mamaria benigna anteriores (Costa et al.,2019). 
Existe um número pequeno de câncer ocasionado por uma predisposição familiar, e dois genes de alto risco foram identificados: BRCA1 e BRCA2. Esses genes, quando mutados, oferecem um maior risco ao desenvolvimento da doença, apesar de acometer uma a cada 1000 mulheres que desenvolvem o câncer antes dos 50 anos de idade. O risco de mulheres sem mutações nesses genes ter neoplasia de mama é 12\%, de 55-65\% quando há mutação no gene BRCA1 e $45 \%$ quando há mutação no gene BRCA2 (Figueiredo et al.,2019).

\section{O mal das senhoras e a mastectomia radical}

As primeiras discussões sobre câncer de mama na medicina brasileira remetem ao início do século XX. Naquele momento, a doença era abordada de forma isolada por alguns médicos, principalmente através de relatos de casos clínicos em sessões de sociedades, como a Sociedade de Medicina e Cirurgia do Rio de Janeiro (Teixeira e Fonseca, 2007). Os tumores dos seios eram considerados comuns em mulheres idosas de classes mais abastadas; embora não houvesse explicação etiológica ou epidemiológica para esse fenômeno, eram vistos como incuráveis, havendo esperança de sobrevida somente através de um procedimento cirúrgico: a mastectomia radical.

No Brasil, as primeiras iniciativas médicas de atuação contra a doença surgiram na década de 1920, mas somente nos anos 1940, no contexto de desenvolvimento de novas tecnologias diagnósticas e terapêuticas e de ampliação da medicina hospitalar, o câncer passou a ser visto como um problema de saúde pública, ou seja, uma doença que deveria ser alvo de ações coordenadas ou executadas por setores do Estado (Teixeira; Fonseca, 2007). Esse processo abrangeu diversos tipos de tumores, principalmente os de maior notabilidade (fosse pela mortalidade elevada ou pela carga simbólica a eles relacionada), mas repercutiu de maneiras distintas entre os vários cânceres. Discutiremos aqui os caminhos que levaram o câncer de mama ao estatuto de importante problema de saúde pública e tópico constante de debates pela sociedade brasileira.

A introdução da mastectomia radical no Brasil foi o grande ponto de virada nas discussões sobre câncer de mama nas primeiras décadas do século XX, principalmente a partir de 1920. Artigos em periódicos especializados (Brazil Médico, Revista Brasileira de Medicina, Archivos Brasileiros de Medicina) e teses das faculdades do Rio de Janeiro e Salvador discutiram os seus resultados, indicando a possibilidade de sobrevida das mulheres entre 3 e 5 anos, nos melhores cenários; um avanço considerável aos olhos dos cirurgiões no período. No entanto, para que as cirurgias tivessem sucesso, era necessário trazer as mulheres para o âmbito dos cuidados médicos, e fazê-lo tão logo a doença estivesse passível de ser percebida. Tal desafio não era de fácil resolução, pois, até o início do século XX, a medicina oficial compartilhava com outros sistemas de cura a preferência da população. No caso do câncer, o problema era agravado pela inexistência de uma terapêutica contra a doença vista como eficaz. Para os médicos, a reticência da população em procurar a ajuda médica resultava em diagnósticos de tumores em estágios bastante avançados. (Teixeira, 2020)

\section{Educação em saúde e o Câncer de mama}

A elevada incidência e mortalidade pelo câncer de mama está relacionada a vários fatores de risco. $\mathrm{O}$ fato de ser do gênero feminino já é considerado um fator de risco para o surgimento do câncer de mama, em razão da quantidade de tecido mamário exposta ao estrogênio. Além disso, fatores como idade, obesidade e exposição ao tabaco também são considerados riscos para desenvolver essa neoplasia. De outra maneira, enfatiza-se que os fatores hereditários correspondem a somente 5\% dos casos, exceto quando o carcinoma é diagnosticado em mulheres jovens, responsável por $25 \%$ dos casos. Portanto, a maioria das condições de risco é de origem ambiental, o que implica adotar medidas simples de prevenção de caráter primário como mudanças no estilo de vida, atividade física regular, alimentação saudável e manutenção do peso, as quais podem reduzir em 30\% os riscos dessa neoplasia (Prado et al., 2020; Santos et al., 2021). 
Nesse contexto, a educação em saúde torna-se fundamental e enriquecedora ao abranger o conhecimento sobre informações dos fatores de risco do câncer de mama, tendo o intuito de promover a prevenção, estímulo ao tratamento e o aumento de sobrevida em mulheres. De outro modo, no tocante aos profissionais que desenvolvem estratégias de educação em saúde no âmbito assistencial surge o ilustre papel do enfermeiro, cujo dever é destinado ao cuidado holístico dentro da atenção básica, na ótica da proteção dos agravos de saúde, por mediar ações de promoção, prevenção e proteção à saúde (Nunes et al., 2020; Cunha et al., 2018).

\section{Câncer de mama e o papel do enfermeiro}

Um conhecimento sólido e consistente reflete de forma positiva na atitude e prática profissional do enfermeiro. Assim, o enfermeiro pode e deve desenvolver práticas voltadas para a prevenção do câncer de mama e promoção da saúde da população adscrita, como grupos de discussões, oficinas, sala de espera, dentre outras atividades que emponderem as usuárias sobre o câncer de mama. Na Consulta de Enfermagem, uma ferramenta efetiva e respaldada por lei, o enfermeiro tem um espaço oportuno para a realização do diagnóstico, a detecção precoce, o tratamento de doenças e a prevenção de condições evitáveis. O enfermeiro é um profissional dotado da capacidade reflexiva-crítica e humanizada, embasado no teor científico e intelectual, capaz de intervir nas situações e nos problemas de saúde/doenças mais prevalentes no perfil epidemiológico nacional (Costa et al.,2019).

É relevante que o enfermeiro desenvolva ações para o enfrentamento à neoplasia mamária, conheça os métodos de detecção precoce e realize ações de rastreamento para identificação antecedente do câncer de mama ou de lesões precursoras em indivíduos assintomáticos o mais rápido possível, para que sejam implementadas medidas efetivas reduzindo sua mortalidade. (Ferreira et al.,2019). A elevação das taxas de incidência e prevalência do câncer de mama indica a necessidade de conhecer a atuação dos profissionais de enfermagem frente ao câncer de mama (Carvalho et al.,2019).

\section{Qualidade de vida e câncer de mama}

Esta doença está intensificando-se na sociedade devido ao processo de transição sociodemográfica de doenças infecciosas para crônico-degenerativas, aumento do índice de desenvolvimento humano, mudanças no estilo de vida, hábitos alimentares, avanços na área da saúde e medicina, dentre outros fatores que prolongam a expectativa de vida, mas não contribuem para a redução aos estímulos agressores para o desenvolvimento do câncer, como envelhecimento, exposição a agentes cancerígenos, obesidade, stress, e outros (Ferreira et al.,2019).

Considerando a alta incidência e o impacto que o diagnóstico e o tratamento do câncer de mama acarretam na vida da mulher, os estudos têm ressaltado a importância de pesquisar sobre a qualidade de vida relacionada à saúde. A qualidade de vida (QV) é definida pela Organização Mundial da Saúde (OMS) como a percepção que o indivíduo tem de si mesmo, da sua posição na vida dentro do contexto de cultura e sistema de valores nos quais ele vive e em relação às suas metas, expectativas e padrões sociais (Ferreira et al.,2019).

A busca da qualidade de vida e de sobrevida livre da doença tem sido cada vez mais importante para o sucesso da terapêutica. A QV pode ser mensurada em pacientes oncológicos com o intuito de medir o impacto da doença na vida do indivíduo. Essa mensuração pode ser feita através de questionários que são capazes de mensurar o problema, favorecendo a detecção de necessidades funcionais como fatores psicológicos e sociais dos pacientes, além de ser utilizada como indicador para nortear estratégias de intervenções terapêuticas e planejamento de ações para promoção de saúde individual ou coletiva (Ferreira et al.,2019). 


\section{Câncer de mama e Covid-19}

As tentativas de conter a Covid-19 forçaram os países a aderirem ao isolamento e ao distanciamento social. As mulheres com diagnóstico de câncer de mama são consideradas uma população vulnerável e apresentam sintomas graves causados pela Covid-194. Entretanto, o distanciamento social e a interrupção das atividades realizadas, como a prática de exercício físico e/ou fisioterapia, podem ter um impacto negativo na saúde global dessas mulheres. Parte do desafio neste momento é continuar a fornecer os cuidados clínicos necessários de uma maneira segura tanto para os profissionais da saúde quanto para essa população (Lyra1 et al.,2021).

Sendo assim, a opção atual são os atendimentos remotos, por serem seguros, criando-se estratégias para a manutenção da saúde dessas mulheres ${ }^{3}$. A literatura evidencia, por meio de uma metanálise, que a atividade física é essencial a essa população e pode reduzir a recorrência do câncer de mama e ainda minimizar os riscos de todas as causas de morte provocados pela doença5; além disso, a Sociedade Americana de Câncer recomenda a atividade física para a prevenção do câncer6; ou seja, em um momento em que não é possível ter-se atividade física presencial, novas estratégias são necessárias, como, por exemplo, aulas remotas diárias, uma vez que ficar sem a atividade física não é uma possibilidade. Sendo assim, o objetivo deste estudo é investigar a prática de atividade física remota e suas implicações durante isolamento social imposto pela pandemia da Covid-19 em mulheres com câncer de mama (Lyra1 et al.,2021).

\section{Conclusão}

Neste artigo, discutimos assistência do enfermeiro diante do câncer de mama na estratégia da família e da saúde pública sobre o câncer de mama no Brasil ao longo do século XX.

Sendo fundamental a ação do enfermeiro durante as consultas na Atenção à Saúde da Mulher na detecção destas anormalidades através do acolhimento, no exame clínico das mamas, na educação em saúde e solicitando exames mais complexos quando necessário tendo consciência que os cuidados a mulheres não devem estar somente no outubro rosa.

Entende-se que, essa neoplasia ocasiona muitas fragilidades para as mulheres acometidas, por diversos fatores, principalmente pelo fato de uma das modalidades terapêuticas para este tipo de câncer ser a cirurgia de mastectomia considerada ainda como o principal recurso para melhorar, controlar e erradicar a doença.

Pode-se concluir com a realização do trabalho que o conhecimento e cuidados dos enfermeiros é essencial na trajetória, isto pode facilitar a detecção precoce dos casos de câncer de mama. Também é importante o enfoque da prevenção primária na ESF em relação a educar as mulheres para a realização de exames periódicos que permitam o diagnóstico precoce, doença esta responsável pela morte de inúmeras mulheres brasileiras que deixaram a saúde ginecológica de lado.

A reflexão tem por sua finalidade, trazer a importância vinculada a novas pesquisas e estudos com a temática assistência do enfermeiro diante do câncer de mama na estratégia da família. Tendo por seu papel principal, estratégias e medidas traçadas acerca da necessidade de um plano e ações de cuidados diferenciados, colocando em pauta a adequação e complexidade do paciente. Relevando também um bom conhecimento e as potenciais complicações no decorrer do tratamento, abordando da melhor forma com qualidade e agilidade, mantendo-se sempre como base o bem estar.

\section{Referências}

Almeida, M. M. M., Almeida, P. F. D., \& Melo, E. A. (2020). Regulação assistencial ou cada um por si? Lições a partir da detecção precoce do câncer de mama em redes regionalizadas do Sistema Único de Saúde (SUS). Interface-Comunicação, Saúde, Educação, 24 https://doi.org/10.1590/Interface.190609.

Amaral, L. B. D. M. X. (2020). Cuidado de enfermagem nos efeitos adversos na quimioterapia para câncer de mama: revisão narrativa da literatura http://repositorio.uft.edu.br/handle/11612/1594

Assis, M. D., Santos, R. O. M. D., \& Migowski, A. (2020). Detecção precoce do câncer de mama na mídia brasileira no Outubro Rosa. Physis: Revista de Saúde Coletiva, 30 https://doi.org/10.1590/S0103-73312020300119. 
Coelho, L. A. C., Lopes, L. S., Bittencourt, M. C., Pereira, A. J. A., Panzetti, T. M. N., Costa, B. N. T., \& Magno, J. V. E. P. (2021). Educação em saúde na prevenção ao câncer de mama em uma Estratégia Saúde da Família em Belém-PA. Research, Society and Development, 10(4), e12910413810-e12910413810 https://doi.org/10.33448/rsd-v10i4.13810.

da Costa Viegas, A., Muniz, R. M., Cardoso, D. H., dos Santos, B. P., Machado, J. B., \& Lindemann, L. G. (2019). Prevenção do câncer de mama: conhecimento de mulheres de uma unidade saúde da família. Saúde em Revista, 19(51), 57-76 https://doi.org/10.15600/2238-1244/sr.v19n51p57-76.

da Silva, D. L., de Oliveira, G. S. C., da Silva Pires, P. R., dos Santos Reis, R. V., de Souza, R. H., da Silva Soeiro, V. M., \& da Silva Viana, L. (2021). Evidências para a assistência de enfermagem à gestante com câncer de mama: revisão integrativa. Saúde Coletiva (Barueri), 11(65), 6066-6079 https://doi. org/10.36489/saudecoletiva.2021v11i65p6066-6079.

de Cavatá Souza, T., da Rosa Monteiro, D., Trevisan, B. F., \& Mallmann, F. H. (2020). Atuação da enfermagem no cuidado a pacientes com câncer de mama: revisão integrativa. Research, Society and Development, 9(12), e14391210939-e14391210939 https://doi.org/10.33448/rsd-v9i12.10939.

Feitosa, E. M., de Sá, M. A. P., da Silva Andrade, E. G., \& dos Santos, W. L. (2018). Assistência de enfermagem no rastreamento do câncer de mama. Revista JRG de Estudos Acadêmicos, 1(3), 27-35 https://doi.org/10.5281/zenodo.4322025.

Ferreira, D. D. S., Bernardo, F. M. D. S., Costa, E. C., Maciel, N. D. S., Costa, R. L. D., \& Carvalho, C. M. D. L. (2020). Conhecimento, atitude e prática de enfermeiros na detecção do câncer de mama. Escola Anna Nery, 24 https://doi.org/10.1590/2177-9465-EAN-2019-0054.

Inacio, D., \& Venson, F. D. Cuidados de enfermagem à pessoa com câncer de mama em unidade de internação e ambulatório hospitalar http://repositorio.unesc.net/handle/1/8651.

Leite, A. C., Silva, M. P. B., Alves, R. S. S., de Lima Silva, M., Almeida, D. S., Feitosa, L. M. H., ... \& Sobrinho, W. D. (2021). Assistência de enfermagem no rastreamento do câncer de mama em pacientes atendidas na Unidade Básica de Saúde. Research, Society and Development, 10(1), e8510111464e8510111464 https://doi.org/10.33448/rsd-v10i1.11464.

Lyra, V. B., de Bem Fretta, T., Stein, F., Sperandio, F. F., \& de Azevedo Guimarães, A. C. (2021). Câncer de Mama e Atividade Física: Percepções durante a Pandemia de Covid-19. Revista Brasileira de Cancerologia, 67(2) https://doi.org/10.32635/2176-9745.RBC.2021v67n2.1291.

Marques, C. A. V., de Gutiérrez, M. G. R., \& de Figueiredo, E. N. (2015). Políticas de saúde pública para o controle do câncer de mama no Brasil [Public health policies for breast cancer control in Brazil]. Revista Enfermagem UERJ, 23(2), 272-278 http://dx.doi.org/10.12957/reuerj.2015.13632.

Oliveira, A. L. R., Michelini, F. S., Spada, F. C., Pires, K. G., de Oliveira Costa, L., de Figueiredo, S. B. C., \& Lemos, A. (2020). Fatores de risco e prevenção do câncer de mama. Cadernos da Medicina-UNIFESO, 2(3).

Ramos, R. S. (2020). Ações publicas para prevenção do câncer de mama no Brasil: revisão de literatura.

Ribeiro, W. A., da Silva, A. C. V., \& da Silva Evangelista, D. (2020). Câncer de mama masculino: contributos do enfermeiro na atenção primária de saúde. Revista Pró-univerSUS, 11(1), 65-73 https://doi.org/10.21727/rpu.v11i1.2291.

Rodrigues, J. R. G. et al. (2020). Importância do enfermeiro para o controle do câncer de mama: revisão narrativa. Revista Eletrônica Acervo Saúde, (55), e3668-e3668 https://doi.org/10.25248/reas.e3668.2020.

Silva, R. R. D. et al. (2021). Ações do enfermeiro para prevenção e detecção precoce do câncer de mama. Saúde Coletiva (Barueri), 11(65), 6090-6099 https://doi.org/10.36489/saudecoletiva.2021v11i65p6090-6099.

Teixeira, L. A., \& Araújo, L. A. (2020). Câncer de mama no Brasil: medicina e saúde pública no século XX. Saúde e Sociedade, 29 https://doi.org/10.1590/S0104-12902020180753.

Traldi, M. C., Galvão, P., Morais, S. S. D., \& Fonseca, M. R. C. D. C. (2016). Demora no diagnóstico de câncer de mama de mulheres atendidas no Sistema Público de Saúde. Cadernos Saúde Coletiva, 24, 185-191 https://doi.org/10.1590/1414-462X201600020026. 